\title{
Severe hepatic toxicity after administration of infliximab in psoriasis treatment
}

\author{
Oana Gabriela Chiriac ${ }^{1}$, Anca Trifan ${ }^{1,2}$, Ana Maria Singeap ${ }^{1,2}$, Camelia Cojocariu ${ }^{1,2}$, \\ Carol Stanciu ${ }^{1,2}$, Catalin Sfarti ${ }^{1,2,}$ \\ ${ }^{1}$ Grigore T. Popa" University of Medicine and Pharmacy, lasi, Romania, ${ }^{2}$ Gastroenterology and \\ Hepatology Clinic, "Sf. Spiridon" Clinical Emergency Hospital, Iasi, Romania
}

\begin{abstract}
The true incidence of drug-induced liver injury (DILI) is difficult to discern because of an unknown denominator of individuals receiving a drug, lack of a simple objective test for the diagnosis of DILI, lack of consensus on what liver test abnormalities constitute DILI, difficulty in attribution of causation to a single drug in those on many medicines, and lack of systematic reporting. Over 1000 medications and herbal products have been involved in causing liver injury, and the list continues to grow. Regarding the biologic therapy with infliximab, according to specialized studies, usually there were mild to moderate elevations of liver enzymes. Hereby, we present a clinical case of drug-induced hepatitis, after the administration of monoclonal antibodies over six months, with a remarkable contrast between the major biochemical changes and the absence of any symptoms.
\end{abstract}

Keywords: biologic therapy, infliximab, psoriasis, liver injury, DILI

\section{Introduction}

Over 1000 medications and herbal products have been involved in causing liver injury etiology, and the list continues to grow. Drug toxicity is the most common cause of acute liver failure, representing an important diagnostic and therapeutic challenge for physicians [1, 2]. Regarding the biologic therapy with infliximab, according to specialized studies, usually there were mild to moderate elevations in ALT, AST (5 times over normal).

Therefore, we present a clinical case of drug-induced hepatitis, after the administration of monoclonal antibodies over six months, with

Received: September 2014; Accepted after review: October 2014; Published: December 2014

*Corresponding author: Catalin Sfarti, MD, PhD, Gastroenterology and Hepatology Clinic, "Sf. Spiridon" Clinical Emergency Hospital, 1 Independenței Boulevard, lași, Romania. Email: cvsfarti@gmail.com a remarkable contrast between the major biochemical changes (transaminases approximately 100 times over normal - one of the few cases reported in the literature with such an important increase) and the absence of any symptoms.

\section{Case report}

A 37 year-old patient being an inactive HBV carrier, diagnosed with psoriasis vulgaris, with no response to standard treatment (stress reduction, climate-therapy, properly moisturizing, topical and systemic therapy with corticosteroids, immunomodulators), for which was initiated the biologic therapy with infliximab in May 2013, is admitted to our clinic in November 2013, directed by his dermatologist for investigations and specialized therapy due to a hepatocytolysis 
syndrome $\quad(A S T=901 \quad \mathrm{U} / \mathrm{l}, \quad \mathrm{ALT}=672 \mathrm{U} / \mathrm{l})$, installed after taking the medication. It should be noted that after each previous administration of Infliximab, the patient had mild and transient increases in liver function tests. Physical examination: at the moment of admission the patient was in dermatological remission, facial erythrosis was found, otherwise without any changes. The first laboratory test had shown hepatocytolysis (ALT=1960 U/l, AST=2301 U/l), cholestasis (GGT=782U/l, FA=467 U/l, DBIL=3.91 mg/dl, TBIL $=5.3 \mathrm{mg} / \mathrm{dl}$ ) and dyslipidemia. It is important to know that no specific treatment is indicated for drug-induced hepatic disease, and that the first step is to discontinue the suspected drug, fact that was already done by the dermatologist. Given the fact that the patient was completely asymptomatic, only a supportive treatment was established right from the first day (liver protection drugs). Despite all of this measures, during the hospitalization, a biological profile corresponding to acute liver dysfunction was outlined, with progressive increments of the transaminases, until a maximum of about 2935 $\mathrm{U} / \mathrm{I}(\mathrm{ALT})$ and $2417 \mathrm{U} / \mathrm{I}$ (AST), in only 5 days from admission, maintaining the significant and also increased cholestasis (GGT $=843 \mathrm{U} / \mathrm{L}$, $\mathrm{FA}=565 \mathrm{U} / \mathrm{L}, \quad \mathrm{DBIL}=5.49 \mathrm{mg} / \mathrm{dl}, \mathrm{TBIL}=7.71$ $\mathrm{mg} / \mathrm{dl}$ ). The management consisted primarily in the exclusion of other causes of liver injury/cholestasis such as viral etiology (antibodies IgM antiHAV or a possible reactivation of $\mathrm{HBV}$ ) other toxic, autoimmune hepatitis, Wilson disease, hemochromatosis, alpha1-antitrypsin deficiency, shock liver, acute cholecystitis, cholangitis, Budd-Chiari syndrome, alcoholic liver disease, malignancy, coagulation disorders [3].

The abdominal ultrasonography described steatosis, and any pathology of the gall bladder, bile ducts, or hepatic tumors were excluded. Histopathologic evaluation remains an important tool in diagnosis. A liver biopsy is not essential, like in this case, especially if we balance the risk and benefits, but a morphologic pattern consistent with the expected diagnosis provides supportive evidence.

The known data from literature about infliximab induced hepatitis shows only mild- moderate elevations of transaminases in contrast with lab values from our patient. It is important to specify that we had contacted the producing company of the drug, asking if they had knowledge about similar cases. According to their experience in clinical trials, regarding the hepatobiliary events, there have been mild to moderate increases in the values of ALT and AST in patients who used infliximab, without progression to severe liver disease. Most abnormal aminotransferase values were transient. In general, patients who experienced increases in ALT and AST were asymptomatic, and these abnormal values have declined or disappeared from stopping or continuing treatment with infliximab or modification of concomitant therapies. In aftermarket studies, have been rarely reported cases of jaundice and non-infectious hepatitis, some with autoimmune hepatitis $[4,5]$. However, there were isolated cases of liver failure which led to liver transplantation or death. Patients with symptoms or signs of liver dysfunction should be evaluated for evidence of liver damage. If jaundice and /or increases in $A L T \geq 5$ times above the upper limit of normal values are detected, the treatment must be interrupted and a thorough investigation should be made of these abnormalities.

In our case, as you have seen above, we performed test to exclude other possible etiologies and the negative results of viral markers, allowed us to initiate the corticosteroid therapy (metilprednisolon i.v.), with fluctuating evolution in the beginning and slow biochemical improvement afterward. After seven days of corticotherapy we had an important regression of the liver test $(A L T=433$ $\mathrm{U} / \mathrm{L}, \mathrm{ASTY}=203, \mathrm{DBIL}=1.29 \mathrm{mg} / \mathrm{dl}$, TBIL=2.12 $\mathrm{mg} / \mathrm{dl})$, and a normal biological profile at one month reassessment.

\section{Discussion}

Drug-induced liver injury (DILI) has an estimated annual incidence between 10 and 15 per 10,000 to 100,000 persons exposed to prescription medications. DILI accounts for approximately 10 percent of all cases of acute hepatitis, and it is also the most frequently 
cited reason for withdrawal of medications from the market place [6].

The aim of this work consists in interdisciplinary collaboration emphasis associated with a proper medical information regarding the administration of a biological therapy, monitoring the response to treatment and declaring the side effects to the pharmacovigilance service of manufacturing companies, because DILI may not be detected prior to drug approval (most new drugs are tested in fewer than 3000 people prior to drug approval). As a result, cases of DILI with an incidence of 1 in 10,000 may be missed. It has been suggested that for every 10 cases of alanine aminotransferase elevation ( $>10$ times the upper limit of normal) in a clinical trial, there will be one case of more severe liver injury that develops once the drug is widely available [7].

The liver is a particular target for drug toxicity because of its role in clearing and metabolizing chemicals. Adverse hepatic events caused by drugs can be considered to be either predictable (high incidence) or unpredictable (low incidence). Drugs that produce predictable liver injury, such as paracetamol, usually do so within a few days and are generally a result of direct liver toxicity of the parent drug or its metabolites. Unpredictable events manifest as overt or symptomatic disease and can occur with intermediate (1-8 weeks) or long (1 year) periods of latency. The majority of adverse drug-induced hepatic events is unpredictable and is either immune-mediated hypersensitivity reactions or are idiosyncratic. The pathogenesis of drug-induced liver injury usually involves the participation of a toxic drug or metabolite that either elicits an immune response or directly affects the biochemistry of the cell. In either case, the resultant cell death is the event that leads to the clinical manifestation of hepatitis. Most instances of drug-induced liver disease are unpredictable and symptoms occur either with intermediate or long periods of latency before onset. Low-frequency, unpredictable reactions, either immune-mediated hypersensitivity or idiosyncratic, often occur on a background of a higher incidence of mild, asymptomatic, and usually transient liver injury [8]. The physician is therefore able to assess the risk of the druginduced liver disease by taking into account the signature of the disease, the latency period, the patient's risk factors, and the exclusion of concomitant drugs and other possible causes. The risk of developing hepatotoxicity involves a complex interplay between the chemical properties of the drug, environmental factors (e.g., the use of concomitant drugs or alcohol), age, sex, underlying diseases (e.g., HIV or diabetes), and genetic factors. The most extensively documented risk factors are concomitant drug use and known hepatitis. The mechanism is not understood, but the effect may be caused by the slow development of an immune response to the drug, combined with its prolonged retention in the body [7, 9].

Management should include the cessation of treatment with the drug, possibly, a short course of high-dose corticosteroids, if the systemic features of hypersensitivity are severe and the withdrawal of cross-reacting drugs (e.g. anticonvulsants and halogenated anesthetics). In all cases of drug-induced liver disease, it is pertinent to assess whether the adverse reaction has been noted previously, is alleviated by the discontinuation of the drug, or recurs if the drug is reintroduced. Also, it is necessary to ensure that other potential causes of the adverse reaction have been excluded. The early identification of an adverse event, together with effective assessment and monitoring, can prevent the occurrence of irreversible liver damage [7].

Drug-induced liver diseases mimic all forms of acute and chronic hepatobiliary diseases. Most drugs with predictable liver toxicity are screened out during preclinical drug development, but unpredictable and rare hypersensitivity or idiosyncratic reactions are often not noted until a drug is used in the clinical situation. Hepatotoxicity caused by drugs, in particular idiosyncratic reactions, is a major challenge to the pharmaceutical industry and physicians [9]. The application of new technologies, such as pharmacogenomics, toxicogenomics, proteomics, and metabonomics, offers the potential to identify risk factors and clarify the pathogenesis of idiosyncratic hepatotoxicity. These technologies may be useful during drug 
development in predicting trouble during animal-model studies and in the post marketing assessment of idiosyncratic reactions [10].

\section{Conclusions}

In this case it was necessary to contact the farmacovigilence service of the producing company for reporting the case and establish a proper therapeutic conduct, considering the relationship of causality between the severity of liver injury and the treatment with Infliximab being directly related. The particularity of the case lies in the context, in which liver changes were highlighted, with mild and transient increase in transaminases, after each administration of infliximab, and a huge increase at one administration, which ultimately imposed a permanent discontinuation of this medication in the case presented. This progressive toxic effect can be explained by the long half-life of this monoclonal antibody (8 weeks), which favored the accumulation of the drug with an important aggressive effect on the liver, who caused

Authors declare no conflicts of interest.

\section{References}

1. Abboud G, Kaplowitz N. Drug-induced liver injury. Drug Saf 2007; 30:277-294.

2. Leise MD, Poterucha JJ, Talwalkar JA. Drug induced liver injury. Mayo Clinic proceedings. 2014; 89(1):95-106.

3. Tajiri K, Shimizu Y. Practical guidelines for diagnosis and early management of drug induced liver injury. World J Gastroenterol 2008; 14(44):6774-85.

4. Ghabril M, Bonkovsky HL, Kum C, et al. Liver injury from tumor necrosis factor-alpha antagonists: analysis of thirty-four cases. Clin Gastroenterol Hepatol 2013; 11(5):558-564.

5. Grant LM, Rockey DC. Drug induced liver injury. Curr Opin Gastroenterol 2012; 28(3):198-202.

6. Lammert C, Bjornsson E, Niklasson A, Chalasani N. Oral medications with significant ultimately an extremely severe hepatocytolysis syndrome, similar values being described only in a few cases in the literature, according to the pharmacovigilance service report.

It is also important to mention that during hospitalization, the patient was completely asymptomatic, both from the standpoint of liver damage and dermatological pathology. Concerning the long-term evolution of the patient, we emphasize the maintenance of dermatological remission for 6 months after stopping the infliximab therapy.

Unfortunately, after this period, reactivation occurs with skin lesions of psoriasis that raise the suspicion of a pustular type. Waiting for the pathology outcome, the dermatologist was taking into account the need to initiate an immune modulatory treatment with methotrexate if the diagnosis was confirmed. In this reason, the patient was redirected to the Gastroenterology and Hepatology Clinic for reassessment (this time with normal liver tests). In the end, the skin biopsy ruled out the above diagnosis and allowed the establishment of a reserved therapy with topical medications with a favorable evolution so far.

hepatic metabolism at higher risk for hepatic adverse events. Hepatology 2010; 51(2):61520.

7. Chalasani N, Fontana RJ, Bonkovsky HL, et al. Causes, clinical features, and outcomes from a prospective study of drug induced liver injury in the United States. Gastroenterology 2008; 135(6):1924-34.

8. Uetrecht J. Idiosyncratic drug reactions: past, present and future. Chem Res Toxicol 2008; 21(1):84-92.

9. Liss G, Rattan S, Lewis JH. Predicting and preventing acute liver injury. What's new in 2010? Expert Opin Drug Metab Toxicol 2010; 6(9):1047- 61.

10. Bernal, Auzinger G, Dhawan A, Wendon J. Acute liver failure. Lancet 2010; 376(9736):190-201. 